\title{
Pseudo-templated transcription by Escherichia coli RNA polymerase at a mutant promoter
}

\author{
Jean-Philippe Jacques and Miriam M. Susskind ${ }^{1}$ \\ Department of Biological Sciences, University of Southern California, Los Angeles, California 90089-1340 USA
}

\begin{abstract}
$A \mathrm{G} \rightarrow \mathrm{T}$ mutation at the start-point of transcription of the phage P22 sar promoter $(s a r+1 T)$ causes a novel defect in promoter clearance by Escherichia coli RNA polymerase (RNAP) in vitro. Under standard transcription conditions, in the presence of high concentrations of all four NTPs, the predominant products from this promoter are poly $(U)$ chains of varying length. Because the mutation creates a run of four $T$ : A base-pairs from -1 to +3 (TGTT $\rightarrow$ TTTT), we propose that synthesis of poly(U) is pseudo-templated by the $A_{4}$ stretch on the template strand. $\mathbf{G} \rightarrow \bar{A}$ and $G \rightarrow C$ mutations at position +1 do not cause pseudo-templated transcription. Several molecules of poly $(U)$ are produced and released per sar $+1 T$ promoter-polymerase complex without dissociation of RNAP from the template DNA. The exponential relationship between yield and size of individual poly $(U)$ species indicates that there is a constant probability that another $U$ residue will be added to the nascent chain. Presumably, pseudo-templated transcription occurs by a slippage (stuttering) mechanism like that proposed to explain certain kinds of RNA editing in eukaryotic viral mRNAs.
\end{abstract}

[Key Words: E. coli RNA polymerase; pseudo-templated transcription; Sar $+1 T$ ]

Received May 11, 1990; revised version accepted July 31, 1990.

Initiation of transcription by $E$. coli RNA polymerase (RNAP) is a complex process that can be divided into several steps: (1) initial binding of the polymerase holoenzyme (core enzyme $+\sigma$ ) to form an unstable "closed" complex; (2) isomerization to form a stable "open" complex in which the DNA around the start point of transcription is unwound; (3) initiation of RNA synthesis; and (4) promoter clearance, which includes release of the promoter and $\sigma$ subunit, and formation of a stable ternary complex of DNA, nascent RNA, and core enzyme.

Much attention has focused on the early steps leading to formation of the open complex because the rate of open complex formation is clearly a primary determinant of promoter strength and is often the target of control by repressors and activators (for review, see Hoopes and McClure 1987 and Yager and von Hippel 1987). Footprinting studies of open complexes on several different promoters show that the polymerase is in close proximity to the promoter DNA between about -50 and +20 and that the region from -9 to +3 is unwound. Within the protected region are the two stretches of DNA sequence that are most highly conserved among promoters, the -35 and -10 regions. The importance of these sequences is underscored by analyses of promoter-down and promoter-up mutations, most of which cluster in the conserved hexamers $5^{\prime}$ -

${ }^{1}$ Corresponding author.
TTGACA- $3^{\prime}$ and $5^{\prime}$-TATAAT- $3^{\prime}$ and in a few other positions nearby (Fig. 1B). Kinetic studies of several promoters show that these sequences affect the rate parameters for open complex formation (initial binding and/or isomerization).

Recently, biochemical studies on RNA initiation and promoter clearance have revealed that these later steps are more complicated than previously thought. The initiating complex undergoes at least two transitions before forming a mature elongating complex /Carpousis and Gralla 1985; Straney and Crothers 1985, 1987; Levin et al. 1987; Metzger et al. 1989; Krummel and Chamberlin 1989). While the nascent RNA grows to a length of $\sim 10$ nucleotides, the footprint of the ternary complex is very similar to that of the binary open complex; contacts with the upstream promoter sequences are maintained while the downstream (leading) edge of the footprint advances only a few base-pairs. These complexes retain $\sigma$ and usually are unstable; they frequently release the nascent RNA, revert to the binary open complex, and reinitiate another RNA molecule. This repeating cycle of RNA initiation, abortive release, and reinitiation has been observed at all promoters examined by Levin et al. (1987) at low substrate concentrations; for some promoters, abortive cycling is quite pronounced even at optimal substrate concentrations (Carpousis and Gralla 1980; Munson and Reznikoff 1981; Liao et al. 1987). Eventually, the initiating complex escapes from this abortive cycle and undergoes a major transition when 
A

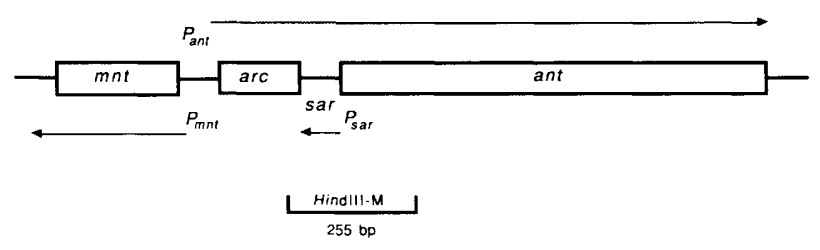

B

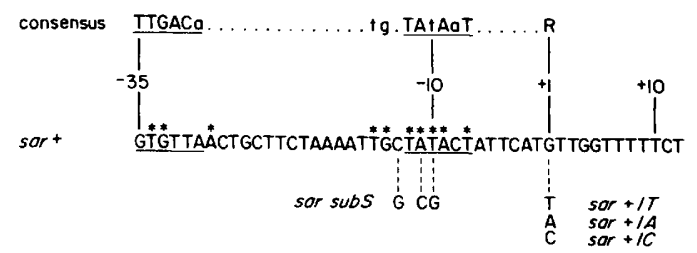

Figure 1. sar and $P_{\text {sar }}(A)$ Map of the $\mathrm{P} 22 \mathrm{immI}$ region (see Wu et al. 1987). Arrows represent the transcripts originating at the respective promoters and $P_{\text {sar. }}$. The location of the HindIII-M fragment used to subclone sar is indicated below. $(B)$ The coding (nontemplate) strand of wild-type $P_{s a r}$ is aligned with a consensus sequence for those positions known to be important by mutational analysis. The most highly conserved bases of the consensus sequence are capitalized. (R) Purine. The -10 and -35 hexamers of both sequences are underlined, and matches between the two are marked by asterisks. Mutations used in this study are shown below the sequence of wild-type $P_{\text {sar }}$.

the RNA is 8-10 nucleotides long: the $\sigma$ factor and upstream region of the promoter are released, the size of the footprint shrinks, and the nascent RNA becomes stably bound. Another reduction in the size of the footprint occurs when the nascent RNA is $\sim 20$ nucleotides long.

Although the rate of open complex formation may be the primary determinant of overall promoter strength in most cases (McClure 1985), there is evidence that for at least some promoters, initiation and promoter clearance can also be rate-limiting. Little is known about the role of the promoter DNA sequence in these processes. Several studies suggest that the same strong contacts with the hexamers that promote rapid formation of open complexes may also exacerbate abortive initiation and inhibit promoter clearance (Carpousis et al. 1982; Kammerer et al. 1986; Knaus and Bujard 1988; Straney and Crothers 1987). Bujard and co-workers have shown that promoter clearance is also affected by the sequence of the transcribed region of the promoter $(+1$ to +20$)$, which can affect overall promoter strength more than 10-fold (Kammerer et al. 1986; Knaus and Bujard 1988). Krummel and Chamberlin (1989) have shown that the stability of initiating ternary complexes is determined by sequences downstream as well as upstream of -1 .

Another peculiar complication during initiation that involves the sequences around the start point has been observed in vitro. At several promoters in which +1 is part of a stretch of three to five identical base-pairs, the polymerase incorporates more copies of the corresponding nucleotide than the template sequence calls for. In the presence of all four nucleoside triphosphates (NTPs), the number of these extra "pseudo-templated" nucleotides varies from 1 to 8 or more, resulting in families of transcripts that have heterogeneous $5^{\prime}$ ends (Machida et al. 1984; Harley et al. 1988, 1990; Guo and Roberts 1990). In all previously reported cases, the polymerase eventually switches to the conventionally templated mode if all four NTPs are present; at some of these promoters, RNAP produces homopolymers if the initiating NTP substrate alone is provided (see Discussion). In this paper, we describe a more extreme case of pseudo-templated transcription from a mutant promoter.

The sar gene of phage P22 encodes a small antisense regulatory RNA that is involved in the immunity circuitry of the phage; sar RNA inhibits synthesis of P22 antirepressor by base-pairing with ant mRNA (Fig. 1A) (Liao et al. 1987; Wu et al. 1987). The sar promoter, $P_{\text {sar }}$, lies within the ant coding sequence. As shown in Figure $1 B$, the -10 and -35 hexamers of $P_{s a r}$ match the consensus sequences at 5 and 3 positions, respectively; the spacer between the hexamers has the consensus length (17 bp) and has the weakly conserved TG sequence at positions -15 and $-14 . P_{s a r}$ is a relatively strong promoter, with high values for the rate constants that govern the kinetics of open complex formation (Wu et al. 1987). However, even under standard transcription conditions, wild-type $P_{\text {sar }}$ produces a large molar excess (15- to 25-fold) of short, abortive products per full-length transcript (Liao et al. 1987). In the course of studies aimed at understanding sar, we found that a $G \rightarrow T$ change at +1 results in a novel phenotype. This mutation, which creates a stretch of four Ts on the coding strand, directs the synthesis of poly|U) "transcripts" of variable length under standard transcription conditions.

\section{Results \\ Sequence requirements for production of an RNA ladder from $\mathrm{P}_{\text {sar }}$}

To gain more insight into the control of ant gene expression by sar RNA, we devised a system for isolating mutations in the sar gene. This system is described briefly here and will be described in detail elsewhere. A singlecopy ant-lacZ gene fusion located on the Salmonella chromosome is fully repressed by wild-type sar RNA provided by a high-copy plasmid, such as pMS390 or pMS610 (Fig. 2). Derivatives of these plasmids carrying mutations in the sar gene or promoter were obtained by mutagenesis with UV light and screening for clones defective in repression of the ant-lacZ fusion. To characterize further some of these mutations, we performed transcription assays in vitro and serendipitously discovered that one mutation, sar $+1 T$ / $\mathrm{a} \mathrm{G} \rightarrow \mathrm{T}$ transversion on the coding strand at position +1 ), has a novel phenotype. 

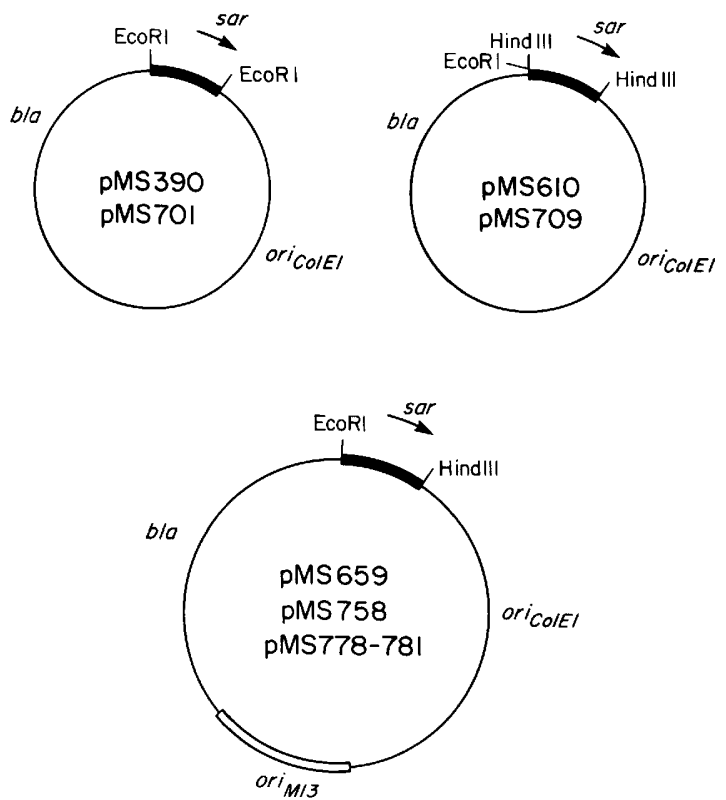

Figure 2. Maps of the sar plasmids. Solid boxes represent the P22 HindIII-M fragment, which extends from -158 to +97 with respect to the $P_{s a r}$ start point. Arrows indicate the direction of sar transcription. Thin lines represent the EcoRI-PvuII backbone of pBR322, and the open box represents the ori ${ }_{\mathrm{M} 13}$ insert in derivatives of $\mathrm{pZ} 152$

Figure 3, lane 1 shows the expected pattern of transcription products labeled with $\left[\alpha-{ }^{32} \mathrm{P}\right] \mathrm{UTP}$ when the assay is performed using the parental $\mathrm{sar}^{+}$plasmid pMS390 as template. The $\mathrm{sar}^{+}$gene produces two major transcripts, which were shown previously by RNA sequence analysis to initiate at $+1 \mathrm{G}$ and to terminate heterogeneously at +68 and +69 (Liao et al. 1987). Surprisingly, the derivative of pMS390 carrying sar $+1 T$ yielded an "RNA ladder" composed of transcripts whose size varies regularly by increments of one (Fig. 3, lane 2). Production of the ladder from the $s a r+1 T$ plasmid did not prevent synthesis of RNA I, which is encoded by the ori $_{\text {ColE1 } 1}$ region of these pBR322 derivatives.

The RNA ladder was reproducibly produced using several independent preparations of the sar $+1 T$ plasmid as template, indicating that the ladder was not caused by RNase contamination. To rule out the idea that contaminating polynucleotide phosphorylase (PNP) might be catalyzing nontemplated polymerization of contaminating $\left[\alpha^{-32} \mathrm{P}\right] \mathrm{UDP}$, we tested whether the reaction was inhibited by either unlabeled UDP $(2-200 \mu \mathrm{M})$ or $\mathrm{K}_{2} \mathrm{HPO}_{4}(800 \mathrm{~mm})$. [Inorganic phosphate displaces the equilibrium towards degradation rather than synthesis of RNA by PNP (McConnell and Bonner 1972).] Neither compound inhibited production of the RNA ladder from the sar $+1 T$ plasmid (data not shown).

In the preliminary experiment shown in Figure 3A, we used RNAP purchased from the Sigma Chemical Co. To challenge these results, the experiment was repeated using RNAP generously provided by the laboratories of C.A. Gross (Univ. of Wisconsin) and M. Chamberlin (Univ. of California, Berkeley). The results shown in

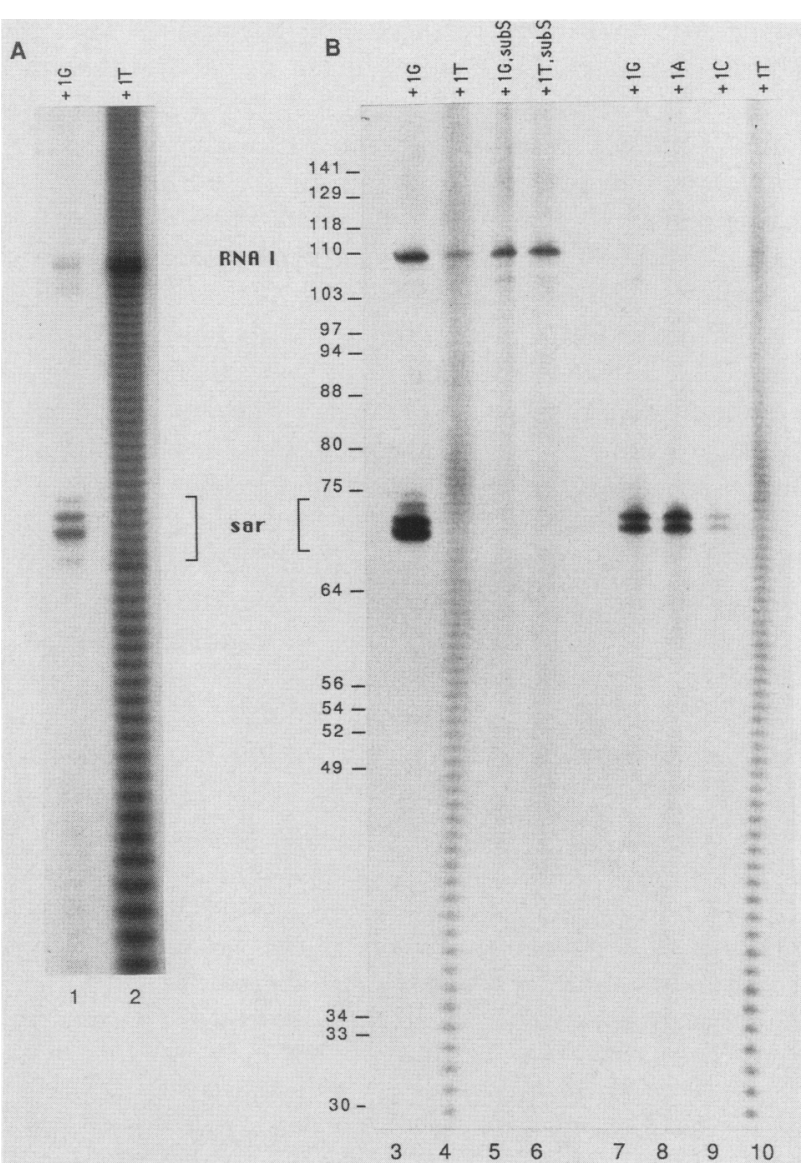

Figure 3. Transcription in vitro of wild-type and mutant sar templates. Transcripts synthesized by RNAP holoenzyme using either supercoiled plasmids (lanes 1-6) or purified restriction fragments (lanes $7-10$ ) as templates were analyzed on a native $8 \%$ acrylamide gel $(A)$ or $8 \%$ acrylamide/7 $\mathrm{M}$ urea gel $(B)$. Transcripts were labeled with $[\alpha-32 \mathrm{P}] \mathrm{UTP}$ under standard conditions in the presence of all 4 NTPs (200 mM each). The positions of the 108-nucleotide ori ${ }_{\text {ColE } 1}$ transcript RNA I and fulllength sar RNA are indicated. The two major bands of sar RNA are 68 and 69 nucleotides in length (Liao et al. 1987). Size markers (not shown) were obtained by running the products of an unrelated ddCTP DNA sequencing reaction in parallel. Template DNAs, whose sar genotypes are indicated at the top of each lane, were: pMS390 (lane 1), pMS701 (lane 2), pMS659 (lane 3), pMS778 (lane 4), pMS758 (lane 5), pMS781 (lane 6), the EcoRI-HindIII fragment of pMS659 (lane 7), the HindIII fragment of pMS709 (lane 8), the EcoRI-HindIII fragment of pMS779 (lane 9), and the EcoRI-HindIII fragment of pMS778 (lane 10).

Figure 3A were reproducible with both enzyme preparations (data not shown). All subsequent experiments shown here were carried out using RNAP prepared by J. Ding in the C.A. Gross laboratory; most of these experiments were also carried out with enzyme from the Chamberlin laboratory, with similar results.

Two approaches were used to address the idea that pMS701, the original, UV-induced sar $+1 T$ plasmid used in Figure $3 \mathrm{~A}$, might carry an undetected mutation outside the sequenced region $(-40$ to +80 with respect to the start point). First, the sar insert in pMS701 was de- 
leted, and the resulting plasmid was transcribed in vitro. The RNA ladder was not produced, indicating that the RNA ladder is encoded by the sar insert. Second, a new $s a r+1 T$ plasmid was constructed by oligonucleotide-directed mutagenesis of the $\mathrm{sar}^{+}$plasmid pMS659 (Fig. 2). Figure $3 \mathrm{~B}$ shows the results obtained when the parental $\mathrm{sar}^{+}$plasmid (lane 3 ) and rebuilt sar $+1 T$ plasmid (lane 4) were transcribed in vitro: the rebuilt sar $+1 T$ plasmid produced the RNA ladder. To show that production of this RNA ladder is dependent on the sar promoter, oligonucleotide-directed mutagenesis was used to construct isogenic plasmids carrying subS, a substitution that inactivates the -10 region of $P_{\text {sar }}$ (Fig. 1). The resulting subs plasmids carrying the wild-type $+1 G$ allele (lane 5) or mutant $+1 T$ allele (lane 6) produced neither sar RNA nor the RNA ladder. Hence, production of the RNA ladder requires both the $\mathrm{G} \rightarrow \mathrm{T}$ change at +1 and the intact -10 region of $P_{\text {sar }}$.

Finally, a transcription experiment was carried out using as templates a set of purified sar fragments carrying all possible genotypes at the +1 position (Fig. 3, lanes $7-10$ ). Whereas the fragment carrying sar $+1 T$ produced the RNA ladder, fragments carrying the sar $+1 \mathrm{~A}$ or $s a r+1 \mathrm{C}$ mutation gave transcripts that comigrate with wild-type sar RNA produced from the sar $+1 G$ fragment. $P_{s a r}$ is the only known promoter on the $\sim 270$-bp fragments used as templates in this experiment.

From these observations, we concluded that a single $\mathrm{G} \rightarrow \mathrm{T}$ transversion at the $P_{\text {sar }}$ start point profoundly changes the pattern of transcription from this promoter, resulting in the production of an RNA ladder under standard transcription conditions using either supercoiled or linear DNA as template. We used the subS mutation to show that the previously characterized sar promoter is required for production of the ladder from the sar $+1 T$ template. In contrast, a $\mathrm{G} \rightarrow \mathrm{A}$ or $\mathrm{G} \rightarrow \mathrm{C}$ change at +1 does not cause production of the RNA ladder. Although the latter mutation apparently decreases promoter strength, neither of them appears to affect the location of the start point of transcription.

\section{The RNA ladder is composed of poly(U)}

Although the experiments in Figure 3 showed that synthesis of the RNA ladder was $P_{s a r}$-dependent, they also showed that the ladder produced from the purified sar $+1 T$ fragment did not stop at the position corresponding to the expected run-off transcript $(97$ nucleotides). In addition, the spacing of bands in the ladder did not show the irregularities normally seen in the spacing of bands of RNA or DNA on sequencing gels. These observations led us to think that synthesis of the ladder was not templated in a classical fashion. To test this hypothesis, we examined the transcription products labeled with $\left.\left[\alpha^{-32}\right]\right\}$ UTP in reactions in which either ATP or CTP or GTP was omitted. In all three cases, production of the ladder was unaffected (data not shown).

To investigate the composition of the ladder produced under standard conditions, $\mathrm{sar}^{+}$and $\mathrm{sar}+1 T$ plasmids were transcribed in the presence of all four NTPs (each at $200 \mu \mathrm{M}$ ), using alternatively each of the four $\left[\alpha^{-32}\right.$ P]NTPs as label (Fig. 4). As expected, wild-type sar RNA produced by the control plasmid was labeled with each $\left[\alpha-{ }^{-32}\right.$ P]NTP (lanes $\left.1-4\right)$. In contrast, the ladder produced by the $s a r+1 T$ plasmid was detected only when $\left[\alpha^{-32}\right]$ UTP was used as label (lanes $6-9$ and $11-14$ ). The darker exposures shown in lanes 11-14 are most informative: lane 14 shows that $\left[\alpha{ }^{-32} \mathrm{P}\right] \mathrm{UTP}$-labeled $s a r+1 T$ products consist of a ladder of regularly spaced bands superimposed on a background "smear." It is also evident that approximately six bands running slightly behind the position of wild-type sar RNA are overrepresented in the ladder. When any of the other three NTPs is used as label (lanes 11-13), the background smear and the bands just behind the position of wild-type sar RNA are observed, but the rest of the bands of the ladder are not. We conclude that (1) the bands of the ladder produced from the $s a r+1 T$ promoter under standard transcription conditions are composed predominantly of polyuridylic acid [poly|U)] of varying lengths, and (2) some incorporation of other nucleotides occurs and contributes to the background smear and bands migrating behind wild-type sar RNA.

Lanes 5 and 10 of Figure 4 show the products of transcription reactions in which $\left[\alpha^{-32}\right.$ P]UTP $(200 \mu \mathrm{M})$ was the only substrate present. Under these conditions, the ladder produced from the $s a r+1 T$ template was even more intense (fourfold) than in the reaction in which

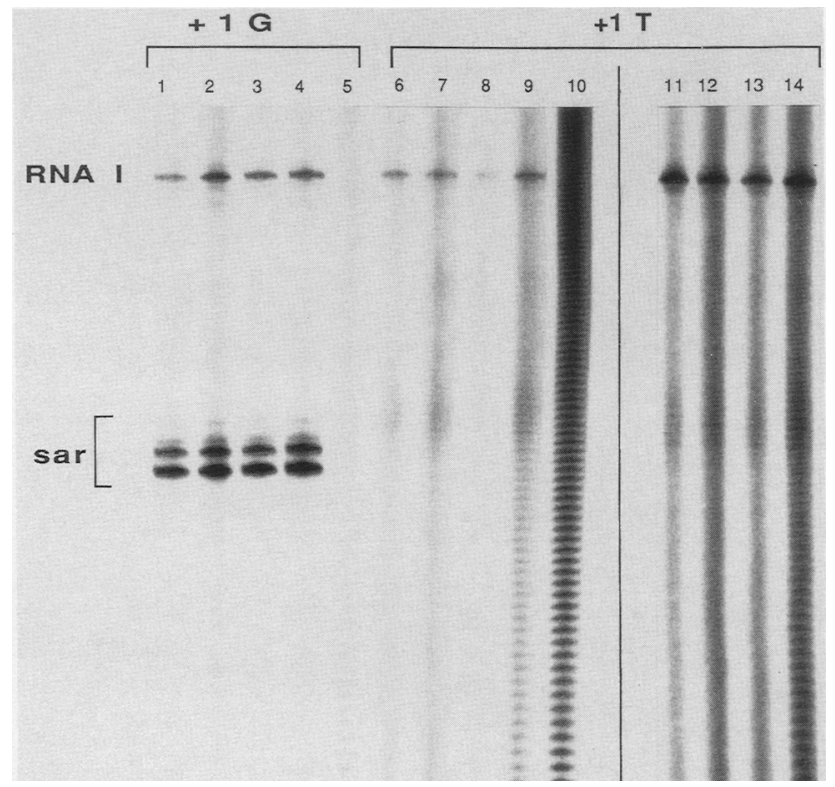

Figure 4. Transcription products labeled with various $\left[\alpha^{-32}\right.$ P]NTPs. Supercoiled plasmids carrying the wild-type (lanes 1-5) or sar $+1 T$ (lanes 6-14) gene (pMS659 and pMS778, respectively) were transcribed in vitro. In lanes 5 and 10, $200 \mu \mathrm{M}$ $\left[\alpha^{-32}\right.$ P $]$ UTP was the only substrate provided. In other lanes, all four NTPs were present at $200 \mu \mathrm{M}$ each, and the label was either $\left[\alpha^{-32}\right.$ P $]$ GTP (lanes 1, 6, and 11), $\left[\alpha^{-32}\right.$ P]ATP (lanes 2, 7, and 12), [ $\alpha^{-32}$ P]CTP (lanes 3,8 , and 13) or [ $\alpha^{-32}$ P]UTP (lanes 4,9 , and 14). Lanes 11-14 are from a longer exposure and a different experiment from lanes $1-10$ 
unlabeled ATP, GTP, and CTP were also present (compare lanes 9 and 10). With $\left[\alpha^{-32}\right.$ P]UTP as the sole substrate, the $\mathrm{sar}^{+}$control plasmid produced a much fainter ladder (cf. lanes 5 and 10). This faint ladder probably did not originate at $P_{\text {sar }}$ because similar results were obtained using the vector plasmid with no sar insert as template (data not shown).

\section{Exponential relationship between abundance and size of $\operatorname{poly}(U)$}

$\left[\gamma^{-32} \mathrm{P}\right] \mathrm{UTP}$, which labels only the $5^{\prime}$ ends of transcripts, was used to measure the relative abundance of transcripts of varying sizes in the RNA ladder produced from the sar $+1 T$ plasmid under standard transcription conditions. The ladder was resolved on $20 \%$ polyacrylamide gels, and the intensities of individual bands were quantified by densitometric scanning of autoradiographs. The semi-logarithmic plots in Figure 5 show that the intensity of each band within the scanned region decreased exponentially as a function of its size. This exponential relationship between abundance and size was observed in the range from 5-mers to the longest transcripts measured (53-mers). It did not apply for dimers, trimers, and 4-mers, which were less abundant than 5-mers (data not shown). The slope of the line varied in different experiments: The ratio of products of length $n$ to products of length $n-1$ was 0.64 for 5 - to 14 -mers (Fig. $5 \mathrm{~A}$ ), 0.80 for 18- to 28 -mers (Fig. 5B), 0.90 for 36- to 46 -mers and 0.91 for 41- to 53-mers (data not shown). (In the ladders shown above, intensity falls off more gradually with size because $\left[\alpha^{-32}\right.$ P]UTP was used as label.) These data imply that once the chain grows to a length of 5 residues, there is a constant probability that another $U$ residue will be added. It is not certain whether the observed variability of this probability is due to day-to-day experimental fluctuation or reflects a true increase in processivity as the nascent chain grows.

RNAP produces several poly(U) molecules without dissociating from the template

The results presented above suggested that $s a r+1 T$ open complexes repeatedly produce poly(U) that is "pseudo-
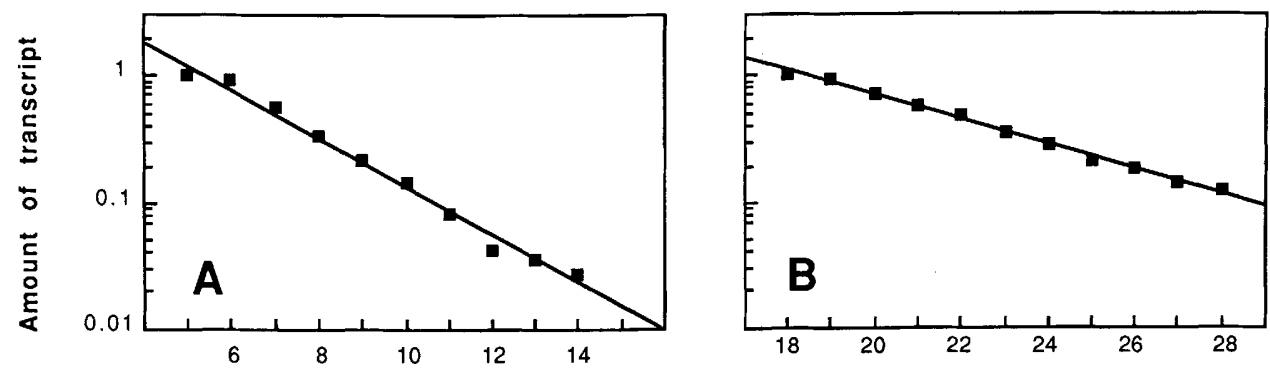

Size of poly(U) (nt)

Figure 5. Exponential relationship between amount and size of poly(U) produced from the sar $+1 T$ promoter. Transcripts were labeled with $\left[\gamma^{-32}\right.$ P $]$ UTP under standard conditions in the presence of all 4 NTPs (200 $\mu \mathrm{M}$ each) using supercoiled pMS778 as template. The relative intensities of sequential bands of the ladder were determined by gel autoradiography and densitometry as described in Methods. 


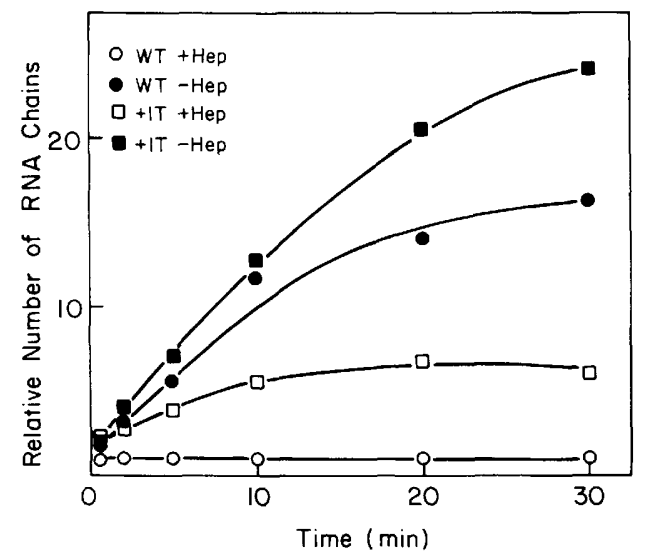

Figure 6. Transcription of wild-type and $s a r+1 T$ templates in the presence or absence of heparin. Template DNAs were preincubated with RNAP for $6 \mathrm{~min}$, heparin (Sigma) was added to half the samples (to $250 \mu \mathrm{g} / \mathrm{ml}$ ), and 3 min later, the reaction was initiated by addition of all four NTPs (to $200 \mu \mathrm{M}$ each, with $\left[\alpha-{ }^{32} \mathrm{P}\right] \mathrm{UTP}$ as label). At various times, the reactions were stopped and transcription products were resolved on a $20 \%$ polyacrylamide gel. Radioactivity in full-length sar RNA or in the 5-mer band of the poly(U) ladder was determined, and the number of RNA chains per template molecule was calculated as described in Methods. Yields are normalized to the value for the $\mathrm{sar}^{+}$template in the presence of heparin 10.31 per DNA molecule/ and are plotted as a function of time after addition of NTPs. Template DNAs were fragments produced by PCR (see Methods). (O) Wild type in the presence of heparin; $(O)$ wild type in the absence of heparin; $(\square)$ sar $+1 T$ in the presence of heparin; $(\mathbf{a})$ sar $+1 T$ in the absence of heparin.

of synthesis and release of poly(U) can occur without dissociation of the polymerase from the sar $+1 T$ template DNA.

\section{Effect of rifampicin on synthesis of poly(U)}

The drug rifampicin inhibits initiation of transcription by RNAP: Formation of the first few phosphodiester bonds is inhibited, so that the only products are dimers $\left(\lambda P_{R}{ }^{\prime}:\right.$ McClure and Cech 1978) or dimers and trimers $\left(P_{\text {lac }}\right.$ UV5: Carpousis and Gralla 1980). However, rifampicin does not inhibit RNA synthesis once the transcription complex is committed to chain elongation (Mangel and Chamberlin 1974; Carpousis and Gralla 1985). Hence, resistance to rifampicin is a characteristic of the elongation complex. To determine the sensitivity of poly $(\mathrm{U})$ synthesis to rifampicin, we performed a series of experiments in which the drug was added either before or after initiation of transcription. First, we examined inhibition of transcription when rifampicin (100 to 500 $\mu \mathrm{M}$ ) was added to preformed open complexes before initiation of the reaction by addition of NTPs. Using either $\mathrm{sar}^{+}$or $\mathrm{sar}+1 \mathrm{~T}$ plasmids as template, no products longer than trimers were produced (data not shown).

We then were interested in knowing whether complexes already actively engaged in poly(U) synthesis are also sensitive to rifampicin. In the experiment shown in Figure 7, preformed open complexes on a sar $+1 T$ tem- plate were treated with heparin, NTPs were added to initiate transcription, and rifampicin was added $3 \mathrm{~min}$ or 8 min later. The results show that synthesis of poly $(\mathbb{U})$ was rapidly and completely inhibited upon addition of rifampicin.

These findings are consistent with the idea that the sar $+1 T$ "idling" complex repeatedly initiates and releases poly(U) chains, and both the original and subsequent initiation events are inhibited by rifampicin. These data do not tell us whether rifampicin blocks elongation of nascent poly|U) chains. Because we are measuring accumulation of labeled products that are made by many rounds of synthesis and release (even in the presence of heparin), a change in the yield of these products during the last round of synthesis would not be detectable.

\section{Discussion}

While studying the mechanism of action of the sar antisense RNA of phage P22, we serendipitously found that a mutant sar promoter with a $\mathrm{G}: \mathrm{C} \rightarrow \mathrm{T}:$ A change at $+1(s a r+1 T)$ shows a novel phenotype during transcription in vitro by $E$. coli RNAP. Under standard conditions, in the presence of all four NTPs, a ladder of regularly spaced bands is seen when radioactive UTP is used as label and the products are resolved on polyacrylamide gels. Because this RNA ladder is seen only if UTP is used as label, and is even more intense if labeled UTP is the only substrate provided, we conclude that these products are poly $(\mathrm{U})$ chains of varying length. Other $P_{\text {sar }}$ mutations at $+1, G: C \rightarrow A: T$ and $G: C \rightarrow C: G$, did not cause this phenotype: These mutant promoters pro-

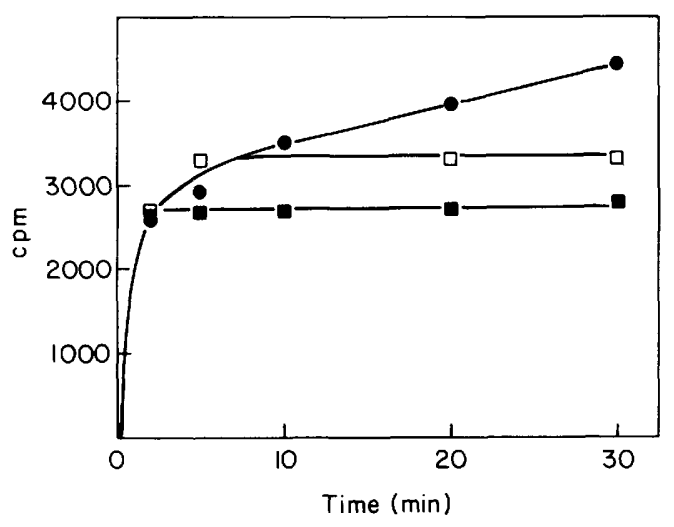

Figure 7. Effect of rifampicin on production of poly(U) from the $s a r+1 T$ promoter. RNAP was incubated with the $s a r+1 T$ plasmid pMS778 for $6 \mathrm{~min}$, heparin was added to $250 \mu \mathrm{g} / \mathrm{ml}$, and 3 min later, the reaction was initiated by addition of NTPs (to $200 \mu \mathrm{M}$ each, with $\left[\alpha^{-32} \mathrm{P}\right] \mathrm{UTP}$ as label). Rifampicin was added after $3 \mathrm{~min}$ or $8 \mathrm{~min}$; no rifampicin was added to the control reaction. At various times, the reactions were stopped and transcription products were resolved on a polyacrylamide gel. Radioactivity in 24 bands of the poly/U) ladder (from 5-mers to 28-mers| was measured as described in Methods and plotted as a function of time after addition of NTPs. (0) sar $+1 T$ in the absence of rifampicin; (D) sar $+1 T$ with rifampicin added at 3 $\min _{i}(\square) s a r+1 T$ with rifampicin added at $8 \mathrm{~min}$. 
duced full-length sar RNAs that comigrate with the wild type.

Because the sar $+1 T$ promoter has a run of four $\mathrm{T}: \mathrm{A}$ base-pairs from -1 to +3 , we hypothesized that open complexes at this promoter produce poly $(\mathrm{U})$ that is pseudo-templated by the stretch of four As on the template strand. Consistent with this idea, synthesis of poly(U) proceeds in the presence of heparin, which traps any polymerases that dissociate from the template DNA (Fig. 6). Because more than five RNA chains are produced per template molecule, we conclude that polymerase remains bound to the $s a r+1 T$ promoter as it repeatedly synthesizes and releases molecules of poly $(\mathrm{U})$. For products in the range from 5-mers to 53-mers, the yield of each poly $(U)$ species decreases exponentially with size, indicating that the polymerase has a constant probability of adding another $U$ residue to the nascent chain. In different experiments, this probability varied from 0.64 to 0.91 . Although this polymerization reaction is much less processive than normal transcription, it is remarkable that such long chains do not dissociate from the ternary complex more readily. Whereas the normal, mature elongation complex includes about $12 \mathrm{bp}$ of RNA-DNA hybrid, no more than four $\mathrm{rU}$ : dA basepairs can occur between poly|U) and the template strand. These considerations are consistent with the idea that the nascent RNA chain is anchored to a site on the enzyme rather than secured simply by base-pairing (Krummel and Chamberlin 1989). Our results also imply that release of the promoter by the enzyme is not triggered by growth of the nascent RNA to a length of $\sim 10$ nucleotides.

Reiterative copying by $E$. coli RNAP was first described more than 25 years ago, when it was shown that poly(A) was produced using denatured, natural DNA or oligo(T) as template and ATP as the sole substrate (see Chamberlin and Berg 1964 and references therein). This phenomenon differed from ours in two important respects: Poly(A) synthesis required a single-stranded template and was severely inhibited by quite low concentrations $(2 \mu \mathrm{M})$ of the other three NTPs. In contrast, we found that sar $+1 T$-promoted poly(U) synthesis was inhibited only about fourfold by high concentrations $(200$ $\mu \mathrm{M}$ ) of the other three NTPs (Fig. 4). Nevertheless, the "slippage" mechanism proposed by Chamberlin and Berg (1964) remains the favored hypothesis to account for reiterative copying (Harley et al. 1990). According to this model, our results could be explained by assuming that the $\mathrm{rU}$ : dA base-pairs between the nascent poly(U) chain and the template DNA strand are transiently broken and reform out of register, such that the RNA chain has slipped back with respect to the DNA chain. This slippage would reexpose one or more of the As in the $\mathrm{A}_{4}$ stretch for use as template for incorporation of another $U$ residue. Chamberlin and Berg (1964) also presented a detailed discussion of the possible reasons for competitive inhibition by other NTPs. Our results do not rule out the related but somewhat more complicated hypothesis that oligo- or poly $(\mathrm{U})$ molecules are released and then rebound and used as primers for synthesis of longer homopolymeric chains. Evidence against this idea has been presented by Harley et al. (1990) in their studies of reiterative copying by $E$. coli RNAP at mutant tet promoters.

Certain others promoters whose start points lie in a stretch of identical base-pairs promote reiterative copying by E. coli RNAP. Nüsslein and Schaller (1975) presented evidence that the phage fd $X$ promoter, which has a $\mathrm{C}_{3}$ stretch in the template strand, produced oligo(G) when GTP was the sole substrate provided. In the presence of all four NTPs, other promoters yield transcripts that are normal except that their $5^{\prime}$ ends have more copies of the initiating nucleotide than are called for by the template sequence. The most clear-cut examples are transcripts that initiate with: oligo(U) pseudo-templated by $\mathrm{A}_{5}$ at the ins $P_{L}$ promoter of IS1 (Machida et al. 1984); oligo(A) pseudo-templated by $\mathrm{T}_{4}$ at two promoters created by insertion of $\mathrm{A}$ : T-rich oligonucleotides in the spacer region of the tet promoter of pBR322 (Harley et al. 1988, 1990); and oligo(A) pseudotemplated by $T_{3}$ at the phage $82 P_{R}^{\prime}$ promoter (Guo and Roberts 1990). In these cases, the number of extra nucleotides at the $5^{\prime}$ end is small, and the polymerase eventually switches from reiterative copying to truly templated transcription. The altered tet promoters also produced oligo(A) with ATP as the sole substrate (Harley et al. 1990). To our knowledge, the $s a r+1 T$ promoter is unique in that it promotes synthesis of long-chain homopolymers in the presence of high concentrations of all four NTPs. The limited reiterative copying seen with the ins $P_{L}$, tet, and $P_{R}{ }^{\prime}$ promoters provides a likely explanation for the slightly overrepresented bands running just behind wild-type sar RNA in the $s a r+1 T$ ladder. Because these bands can be labeled with any of the four NTPs, it is likely that these products result because the polymerase has a tendency to switch to templated transcription after the first few pseudo-templated $U$ residues have been incorporated. However, it is clear that for the sar $+1 T$ promoter, it is more likely that the nascent RNA will slip back or slip away rather than be extended properly.

The promoter clearance step is complicated and poorly understood. Together with studies from other laboratories, our studies of the sar $+1 T$ promoter underscore the importance of steps subsequent to open complex formation. Clearly, promoters differ not only in the rate at which they form open complexes, but also in the productivity of the open complex once formed. Little is known about the determinants that govern the rate of formation of the first few phosphodiester bonds, the tendency to release templated abortive products, the tendency to produce pseudo-templated products, release of the promoter, release of the $\sigma$ subunit, and conversion to a stable elongation complex. "Slippery" promoters have little in common other than the homonucleotide stretch at which slippage presumably occurs. The fact that sar $+1 T$ has a shorter $\mathrm{T}: \mathrm{A}$ stretch than ins $P_{L}$ suggests that some other feature of the sar promoter accentuates reiterative copying. It might be relevant that wild-type $P_{\text {sar }}$ releases an unusually large number of abortive 
products (15-25 per full-length RNA) during transcription initiation under standard conditions (Liao et al. 1987).

Pseudo-templated transcription by a slippage mechanism (also called reiterative copying, stuttering, and chattering) is not unique to E. coli RNAP. This mechanism has been proposed to account for various discrepancies between the sequences of animal viral mRNAs and their respective templates: (1) The poly(A) tails at the $3^{\prime}$ ends of vesicular stomatitis virus mRNAs, which are synthesized by the viral polymerase, are thought to be pseudo-templated by a stretch of 5-7 $U$ residues at the end of each gene on the genomic single-stranded RNA template (Herman et al. 1980; Iverson and Rose 1981). (2) The $5^{\prime}$ ends of vaccinia virus late mRNAs have $\sim 35$ nucleotide poly(A) "heads," which may be pseudotemplated by a $T_{3}$ stretch in the template DNA strand at the transcription start site (Wright and Moss 1987, 1989; Schwer and Stunnenberg 1988). (3) In certain mRNAs of various paramyxoviruses, one (or in some viruses, two) extra $G$ residues are inserted in an internal stretch of three or four Gs within a coding sequence; copies with and without the insertion encode proteins with the same amino-terminal, but different carboxy-terminal, amino acid sequences (Thomas et al. 1988; Cattaneo et al. 1989; Vidal et al. 1990). Pseudo-templated transcription is also reminiscent of proposed mechanisms for generation of frameshift and other types of mutations by misalignment of the template and nascent DNA strands during replication (Kunkel and Soni 1988). At this time, the parameters that determine the propensity of various polymerases to incorporate pseudo-templated nucleotides is unknown.

\section{Methods \\ Plasmids}

Plasmids are described in Table 1 and depicted in Figure 2. The entire $P_{\text {sar }}$-sar region of mutant plasmids was sequenced by the enzymatic method using Sequenase enzyme /U.S. Biochemicals) and plasmid DNA prepared by the alkaline-lysis method (Hattori and Sakaki 1986).

\section{Preparation of DNA templates}

Intact plasmids, purified restriction fragments, or fragments produced by PCR were used as template as indicated in the figure legends. Plasmids were grown in E. coli K12 strain TGl (Amersham) and harvested on a large scale by the method of Clewell (1972) with minor modifications, including a reduced level of chloramphenicol $(40 \mu \mathrm{g} / \mathrm{ml})$. In some cases, plasmid DNA was purified twice rather than once by equilibrium centrifugation in $\mathrm{CsCl}$ density gradients. Restriction fragments carrying $P_{\text {sar }}$ were either the 255-bp HindIII fragment of pMS709 or the 266-bp EcoRI-HindIII fragment of pMS659 and its derivatives. The $5^{\prime}$ end of the nontemplate strand corresponds to -158 or -169 relative to the start point of $P_{s a r}$. These fragments were separated from the plasmid "backbone" $(\geqq 2.3 \mathrm{~kb})$ by $7 \%$ PEG fractionation (Lis and Schleif 1975). The fragments used in Figure 6 were prepared by PCR (Higuchi et al. 1988), using pMS659 and pMS778 as templates and primers selected to generate a 278 -bp fragment extending from -109 to +169 ,
Table 1. Construction of plasmids

\begin{tabular}{|c|c|}
\hline Plasmid & Description $^{\mathbf{a}}$ \\
\hline $\mathrm{pZ} 152$ & $\begin{array}{l}\text { pBR322 derivative in which the smallest DraI } \\
\text { fragment is replaced by a 455-bp ori }{ }_{M 13} \\
\text { fragment (Zagursky and Berman 1984) }\end{array}$ \\
\hline pMS390 & $\begin{array}{l}\text { filled-in HindIII-M sar }{ }^{+} \text {fragment of P22 inserted } \\
\text { in the BamHI (filled-in) site of pMS99, which } \\
\text { flanks the insert with EcoRI sites (Wu et al. } \\
\text { 1987) }\end{array}$ \\
\hline pMS443 & $\begin{array}{l}\text { EcoRI (filled-in)-PvuII bla ori }{ }_{C o l E 1} \text { fragment of } \\
\text { pBR322 joined by one copy of a } 10 \text {-bp HindIII } \\
\text { linker, which recreates the EcoRI site }\end{array}$ \\
\hline pMS610 & $\begin{array}{l}\text { HindIII-M sar }{ }^{+} \text {fragment of P22 inserted in the } \\
\text { HindIII site of pMS443 }\end{array}$ \\
\hline pMS658 & $\begin{array}{l}\text { upstream HindIII site of pMS610 destroyed by } \\
\text { partial digestion with HindIII, filling-in, and } \\
\text { religation }\end{array}$ \\
\hline pMS659 & $\begin{array}{l}\text { AccI-PstI ori }{ }_{M 13} \text { ori }_{C o l E 1} \text { fragment from pZ152 } \\
\text { inserted in place of the corresponding ori }{ }_{C o l E 1} \\
\text { fragment of pMS } 658\end{array}$ \\
\hline pMS701 & sar $+1 T$ mutant of pMS390 (UV induced) \\
\hline pMS709 & sar $+1 A$ mutant of pMS610 (UV induced) \\
\hline pMS758 & subS mutant of pMS659 (site directed) \\
\hline pMS778 & sar $+1 T$ mutant of pMS659 (site directed) \\
\hline pMS779 & sar $+1 C$ mutant of pMS659 (site directed) \\
\hline pMS780 & $\begin{array}{l}\text { sar }+1 C, s u b S \text { mutant of pMS659 (derived from } \\
\text { pMS758 by site-directed mutagenesis) }\end{array}$ \\
\hline pMS781 & $\begin{array}{l}\text { sar }+1 T, \text { subS mutant of pMS659 (derived from } \\
\text { pMS758 by site-directed mutagenesis) }\end{array}$ \\
\hline
\end{tabular}

aFilling-in was done with DNA Poll large fragment. Procedures used to obtain pMS701 and pMS709 by mutagenizing with UV and screening for the $\mathrm{Sar}^{-}$phenotype will be described elsewhere. Site-directed mutagenesis was carried out using the Amersham mutagenesis system, version 2 .

relative to the start point of $P_{s a r}$. The concentration of DNA preparations was determined by measuring the $\mathrm{OD}_{260}$.

\section{Transcription in vitro}

Transcription reactions were carried out in a total volume of 40-60 $\mu$ l containing $20 \mathrm{~mm}$ Tris- $\mathrm{Cl}(\mathrm{pH} 8), 100 \mathrm{mM} \mathrm{KCl}, 10 \mathrm{~mm}$ $\mathrm{MgCl}_{2}, 1 \mathrm{mM}$ dithiothreitol, $100 \mu \mathrm{g} / \mathrm{ml}$ acetylated bovine serum albumin (gift from D. Galas or J. Ding and C.A. Gross), and (unless otherwise noted) $0.6 \mathrm{~nm}$ template DNA and $10 \mathrm{~nm}$ RNAP. Labeled substrates (Amersham) and concentrations of NTPs are given in the figure legends; the sources of RNAP are discussed in Results. Except in the experiments shown in Figures 6 and 7, reactions were initiated by addition of enzyme and terminated after $30 \mathrm{~min}$ at $37^{\circ} \mathrm{C}$ by addition of a $1 / 10$ volume of freshly made stop solution $(50 \%$ glycerol, $0.25 \%$ bromophenol blue, $250 \mathrm{~mm}$ EDTA).

\section{Quantification of transcripts}

Transcripts were resolved by electrophoresis on $7-20 \%$ acrylamide TBE gels without (Fig. 3A only) or with $7 \mathrm{M}$ urea. After drying, gels were autoradiographed using Kodak XAR-5 film. 
Two methods were used to quantify transcription products. In the experiment of Figure 5, the relative yield of transcripts was determined by scanning autoradiographs exposed within the linear range of the film, using a computer-interfaced LKB Ultrascan XL laser densitometer and LKB 2400 Gelscan XL version 1.2 software. In the experiments of Figures 6 and 7 , bands were excised from the gel and radioactivity measured by a liquid scintillation counter. The excised region was either: (1) the two major bands corresponding to full-length wild-type sar RNA; (2) the single band corresponding to the 5 -mer; or $(3)$ a strip containing the bands corresponding to 5 - to 28 -mers, as specified in the figure legends. Molar yields were calculated from the known specific activity of the $\left[\alpha^{-32} \mathrm{P}\right] \mathrm{UTP}$ used, the efficiency of counting, and the number of $U$ residues in the transcript.

\section{Acknowledgments}

We are grateful to M. Chamberlin, J. Ding, and C.A. Gross for generously providing purified $E$. coli RNA polymerase. We also thank M. Chamberlin, D. Galas, W.R. McClure, and C. Harley for helpful discussions and J.W. Roberts for communicating unpublished results. This work was supported by National Institutes of Health grant GM36811 to M.M.S.

The publication costs of this article were defrayed in part by payment of page charges. This article must therefore be hereby marked "advertisement" in accordance with 18 USC section 1734 solely to indicate this fact.

\section{References}

Cattaneo R., K. Kaelin, K. Baczko, and M.A. Billeter. 1989. Measles virus editing provides an additional cysteine-rich protein. Cell 56: 759-764.

Carpousis, A.J. and J.D. Gralla. 1980. Cycling of ribonucleic acid polymerase to produce oligonucleotides during initiation in vitro at the lacUV5 promoter. Biochemistry 19: $3245-3253$.

- 1985. Interaction of RNA polymerase with lacUV5 promoter DNA during mRNA initiation and elongation. J. Mol. Biol. 183: $165-177$.

Carpousis, A.J., J.E. Stephano, and J. Gralla. 1982. 5' Nucleotide heterogeneity and altered initiation of transcription at mutant lac promoters. J. Mol. Biol. 157: 619-633.

Chamberlin, M.J. 1976. In RNA Polymerase (ed. R. Losick and M. Chamberlin), pp. 159-191. Cold Spring Harbor Laboratory, Cold Spring Harbor, N.Y.

Chamberlin, M. and P. Berg. 1964. Mechanism of RNA polymerase action: Characterization of the DNA-dependent synthesis of polyadenylic acid. J. Mol. Biol. 8: 708-726.

Clewall, D.B. 1972. Nature of CoPE1 plasmid replication in Escherichia coli in the presence of chloramphenicol. I. Bacteriol. 110: 667-676.

Guo, H.-C. and J.W. Roberts. 1990. Heterogeneous initiation due to slippage at the bacteriophage 82 late gene promoter in vitro. Biochemistry (in press).

Harley, C.B., J. Lawrie, M. Betlach, H.W. Boyer, and J. Hedgpeth. 1988. Transcription initiation at the tet promoter and effect of mutations. Nucleic Acids Res. 16: 7269-7285.

Harley, C.B., J. Lawrie, H.W. Boyer, and J. Hedgpeth. 1990. Reiterative copying by $E$. coli RNA polymerase during transcription initiation of mutant pBR322 tet promoters. $\mathrm{Nu}$ cleic Acids Res. 18: 547-552.

Hattori, M. and Y. Sakaki. 1986. Dideoxy sequencing method using denatured plasmid templates. Anal. Biochem. 152: $232-238$.

Herman, R.C., M. Schubert, J.D. Keene, and R.A. Lazzarini.
1980. Polycistronic vesicular stomatitis virus RNA transcripts. Proc. Natl. Acad. Sci. 77: 4662-4665.

Higuchi, R., B. Krummel, and R.K. Saiki. 1988. A general method of in vitro preparation and specific mutagenesis of DNA fragments: study of protein and DNA interactions. Nucleic Acids Res. 16: 7351-7367.

Hoopes, B.C. and W.R. McClure. 1987. Strategies in regulation of transcription initiation. In Escherichia coli and Salmonella typhimurium. (ed. F.C. Neidhardt, J.L. Ingraham, K.B. Low, B. Magasanik, and H.E. Umbarger), pp. 1231-1240. American Society for Microbiology, Washington DC.

Iverson, L.E. and J.K. Rose. 1981. Localized attenuation and discontinuous synthesis during vesicular stomatitis virus transcription. Cell 23: 477-484.

Kammerer, W., U. Deuschle, R. Gentz, and H. Bujard. 1986. Functional dissection of $E$. coli promoters: information in the transcribed region is involved in late steps of the overall process. EMBO I. 5: 2995-3000.

Knaus, R. and H. Bujard. 1988. $\mathrm{P}_{\mathrm{L}}$ of coliphage lambda: an alternative solution for an efficient promoter. EMBO J. 7: 29192923.

Krummel, B. and M.J. Chamberlin. 1989. RNA chain initiation by E. coli RNA polymerase. Structural transitions of the enzyme in early ternary complexes. Biochemistry 28: 78297842.

Kunkel, T.A. and A. Soni. 1988. Mutagenesis by transient misalignment. J. Biol. Chem. 263: 14784-14789.

Levin, J.R., B. Krummel, and M.J. Chamberlin. 1987. Isolation and properties of transcribing ternary complexes of $E$. coli RNA polymerase positioned at a single template base. $I$. Mol. Biol. 196: 85-100.

Liao, S.-M., T.-H. Wu, C.H. Chiang, M.M. Susskind, and W.R. McClure. 1987. Control of gene expression in bacteriophage P22 by a small antisense RNA. I: Characterization in vitro of the $P_{s a r}$ promoter and the sar RNA transcript. Genes Dev. 1: 197-203.

Lis, J.T. and R. Schleif. 1975. Size fractionation of doublestranded DNA by precipitation with polyethylene glycol. Nucleic Acids Res. 2: 383-389.

McClure, W.R. 1985. Mechanism and control of transcription initiation in prokaryotes. Annu. Rev. Biochem. 54: 171204.

McClure, W.R. and C.L. Cech. 1978. On the mechanism of rifampicin inhibition of RNA synthesis. I. Biol. Chem. 253: 8949-8956.

McConnell, D.J. and J. Bonner. 1972. Preparation of highly purified ribonucleic acid polymerase; separation from polynucleotide phosphorylase and polyphosphate kinase. Biochemistry 11: 4329-4336.

Machida, C., Y. Machida, and E. Ohtsubo. 1984. Both inverted repeat sequences located at the ends of ISl provide promoter functions. J. Mol. Biol. 177: 247-267.

Mangel, W.F. and M.J. Chamberlin. 1974. Studies of ribonucleic acid chain initiation by Escherichia coli ribonucleic acid polymerase bound to $\mathrm{T} 7$ deoxyribonucleic acid. I. An assay for the rate and extent of ribonucleic acid chain initiation. $J$. Biol. Chem. 249: 2995-3001.

Metzger, W., P. Schickor, and H. Heumann. 1989. A cinematographic view of $E$. coli RNA polymerase translocation. EMBO. I. 8: 2745-2754.

Munson, L.M. and W.S. Reznikoff. 1981. Abortive initiation and long ribonucleic acid synthesis. Biochemistry 20: 2081-2085.

Nüsslein, C. and H. Schaller. 1975. Stabilization of promoter complexes with a single ribonucleoside triphosphate. Eur. I. Biochem. 56: 563-569. 
Schwer, B. and H.G. Stunnenberg. 1988. Vaccinia virus late transcripts generated in vitro have a poly(A) head. EMBO $J$. 7: $1183-1190$.

Straney, D.C. and D.M. Crothers. 1985. Intermediates in transcription initiation from the E. coli lacUV5 promoter. Cell 43: 449-459.

1987. A stressed intermediate in the formation of stably initiated RNA chains at the E. coli lacUV5 promoter. I. Mol. Biol. 193: 267-278.

Thomas S.M., R.A. Lamb, and R.G. Paterson. 1988. Two mRNAs that differ by two nontemplated nucleotides encode the amino coterminal proteins $\mathrm{P}$ and $\mathrm{V}$ of the paramyxovinus SV5. Cell 54: 891-902.

Vidal S., J. Curran, and D. Kolakofsky. 1990. Editing of the Sendai virus $\mathrm{P} / \mathrm{C}$ mRNA by $\mathrm{G}$ insertion occurs during mRNA synthesis via a virus-encoded activity. $/$. Virol. 64: $239-246$.

Wright C.F. and B. Moss. 1987. In vitro synthesis of vaccinia virus late mRNA containing a $5^{\prime}$ poly(A) leader sequence. Proc. Natl. Acad. Sci. 84: 8883-8887.

. 1989. Identifications of factors specific for transcription of the late class of vaccinia virus genes. I. Virol. 63: 42244233.

Wu, T.-H., S.M. Liao, W.R. McClure, and M.M. Susskind. 1987. Control of gene expression in bacteriophage P22 by a small antisense RNA. II: Characterization of mutants defective in repression. Genes Dev. 1: 204-212.

Yager, T.D. and P.H. von Hippel. 1987. Transcript elongation and termination in Escherichia coli. In Escherichia coli and Salmonella typhimurium. (ed. F.C. Neidhardt, J.L. Ingraham, K.B. Low, B. Magasanik, and H.E. Umbarger), pp. 1231-1240. American Society for Microbiology, Washington DC.

Zagursky, R.J. and M.L. Berman. 1984. Cloning vectors that yield high levels of single-stranded DNA for rapid DNA sequencing. Gene 27: 183-191. 


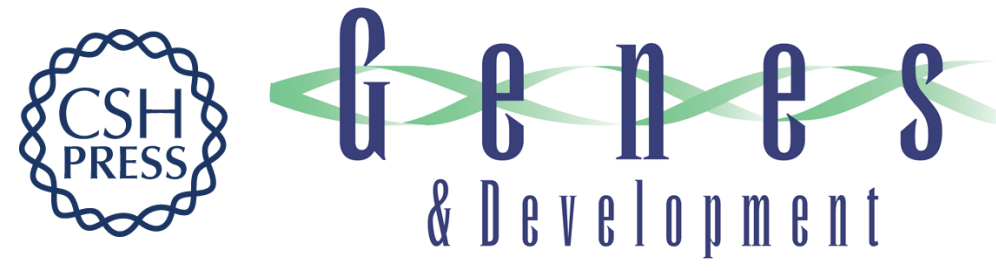

\section{Pseudo-templated transcription by Escherichia coli RNA polymerase at a mutant promoter.}

$\mathrm{J} P$ Jacques and $M \mathrm{M}$ Susskind

Genes Dev. 1990, 4:

Access the most recent version at doi:10.1101/gad.4.10.1801

References This article cites 36 articles, 10 of which can be accessed free at:

http://genesdev.cshlp.org/content/4/10/1801.full.html\#ref-list-1

License

Email Alerting

Service

Receive free email alerts when new articles cite this article - sign up in the box at the top right corner of the article or click here.

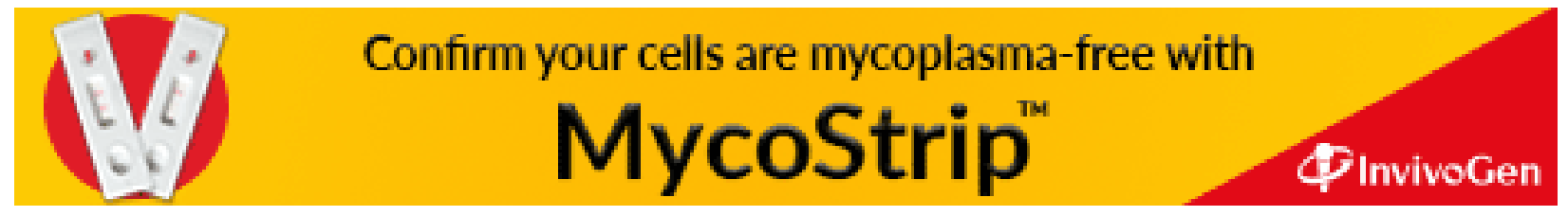

